\title{
MAXIMIZACIÓN DE UTILIDAD Y VALUACIÓN DE OPCIONES CON VOLATILIDAD ESTOCÁSTICA
}

\author{
Francisco Venegas Martínez*
}

Departamento de Contabilidad y Finanzas

Tecnológico de Monterrey, Campus Ciudad de México

Gerardo Pioquinto Aguilar Sánchez

Departamento de Matemáticas

Tecnológico de Monterrey, Campus Ciudad de México

(Recibido 6 de diciembre 2004, aceptado 25 de marzo 2005)

\section{Resumen}

Este trabajo desarrolla un modelo de equilibrio parcial para valuar productos derivados cuando la volatilidad del activo subyacente presenta volatilidad estocástica. El modelo considera una economía poblada por agentes racionales (consumidores-inversionistas) que toman decisiones sobre consumo e inversión en un ambiente de riesgo de mercado. El precio del derivado y el coeficiente de riesgo se caracterizan como las soluciones de un sistema de ecuaciones diferenciales parciales. Varias formas específicas de la función de utilidad son analizadas en el proceso de valuación.

\begin{abstract}
This paper develops a model of partial equilibrium to estimate derivative products when the volatility of the underlying asset displays stochastic volatility. The model considers an economy populated by rational agents (consumer-investors) who make decisions on consumption and investment in an environment of risk market. The price of the derivative and the coeffcient of risk are characterized as solutions of a system of partial differential equations. Several specific forms of the utility function are analyzed in the valuing process.
\end{abstract}

Clasificación JEL: D1, D8, G13

Palabras Clave: Maximización de utilidad, Volatilidad estocástica, Ecuación diferencial parcial

* Tecnológico de Monterrey, Campus Ciudad de México, Calle del Puente 222, Aulas 3, Cuarto piso, Col. Ejidos de Huipulco, Del. Tlalpan, 14380 México, D. F. Correos electrónicos: fvenegas@itesm.mx y gerardo.aguilar@itesm.mx

Los autores están muy agradecidos por los valiosos comentarios de dos dictaminadores anónimos. 


\section{Introducción}

En esta sección se obtiene, a través de agentes racionales maximizadores de utilidad, la ecuación diferencial parcial que caracteriza el precio (valor) de una opción europea de compra cuando la volatilidad es estocástica. En particular, se supone que la volatilidad es conducida por un movimiento geométrico Browniano. Se supone que los agentes tienen acceso a tres activos financieros: una acción, una opción sobre la acción y un bono libre de riesgo de incumplimiento que paga tasa fija. Se supone también que no hay impuestos y que no existen costos de transacción en el mantenimiento del portafolio, es decir, no hay comisiones.

\section{Planteamiento del problema de valuación}

Se supone que el precio del activo subyacente, $S_{t}$, sigue un movimiento geométrico Browniano, cuya volatilidad al cuadrado (la varianza), $\sigma_{t}^{2}=V_{t}$, es conducida por otro movimiento geométrico Browniano, es decir,

$$
\left\{\begin{array}{l}
\mathrm{d} S_{t}=\mu S_{t} \mathrm{~d} t+\sigma_{t} S_{t} \mathrm{~d} W_{t}, \\
\mathrm{~d} V_{t}=\alpha V_{t} \mathrm{~d} t+\beta V_{t} \mathrm{~d} Z_{t},
\end{array}\right.
$$

donde $\mu \in \mathbb{R}$ es el parámetro de tendencia del subyacente, $\alpha \in \mathbb{R}$ es la tendencia de la varianza y $\beta>0$ es la volatilidad de la varianza, las cuales son cantidades conocidas. Asimismo, se supone que los movimientos Brownianos $\mathrm{d} W_{t}$ y $\mathrm{d} Z_{t}$ están correlacionados entre sí, de tal forma que

$$
\operatorname{Cov}\left(\mathrm{d} W_{t}, \mathrm{~d} Z_{t}\right)=p \mathrm{~d} t
$$

Observe, por último, que no se considera el pago de dividendos.

Considere ahora un consumidor-inversionista racional que tiene acceso a tres diferentes activos: una acción de precio $S_{t}$, una opción sobre la acción de precio $c=c\left(S_{i}, V_{t}, t\right)$ y un bono libre de riesgo de incumplimiento que paga una tasa constante $r$. El bono también puede verse como un depósito bancario que paga tasa $r$. Como siempre, se supone que el precio de una opción europea de compra, $c$, depende de las variables de estado, esto es, $c=c\left(S_{t}, V_{t}, t\right)$. En lo subsecuente, $a_{t}$ denotará la riqueza real del agente al instante $t$. Las proporciones de la riqueza que el agente asigna a la tenencia de los diferentes activos, la acción, el derivado y el bono serán denotadas, respectivamente, por $x_{t}, y_{t} \mathrm{y}$ $1-x_{t}-y_{t}$. La ecuación de evolución de la riqueza real (restricción presupuestal) está dada por

$$
\mathrm{d} a_{t}=x_{t} a_{t} \mathrm{~d} R_{S}+y_{t} a_{t} \mathrm{~d} R_{c}+\left(1-x_{t}-y_{t}\right) a_{t} r \mathrm{~d} t-c_{t} \mathrm{~d} t
$$

donde $\mathrm{d} R_{S}=\mathrm{d} S_{t} / S_{t} \mathrm{y} \mathrm{d} R_{c}=\mathrm{d} c / c$. Es importante destacar la diferencia entre c y $c_{t}$, el primer caso se refiere al precio de la opción y el segundo al bien de consumo. Ahora bien, de acuerdo con el lema de Itô, se tiene que el precio de la opción sigue una ecuación de la forma

$$
\mathrm{d} c=\mu_{c} c \mathrm{~d} t+\sigma_{c} c \mathrm{~d} W_{t}+\xi_{c} c \mathrm{~d} Z_{t}
$$


donde los coeficientes $\mu_{c}, \sigma_{c}$ y $\xi_{c}$ están dados, respectivamente, por:

$$
\begin{gathered}
\mu_{c}=\frac{1}{c}\left(\frac{\partial c}{\partial t}+\mu S_{t} \frac{\partial c}{\partial S_{t}}+\alpha V_{t} \frac{\partial c}{\partial V_{t}}+\frac{1}{2} \sigma_{t}{ }^{2} S_{t}{ }^{2} \frac{\partial^{2} c}{\partial S_{t}{ }^{2}}\right. \\
\left.+\frac{1}{2} \beta^{2} V_{t}^{2} \frac{\partial^{2} c}{\partial V_{t}^{2}}+\rho \beta V_{t} \sigma_{t} S_{t} \frac{\partial^{2} c}{\partial S_{t} \partial V_{t}}\right), \\
\sigma_{c}=\frac{1}{c} \sigma_{t} S_{t} \frac{\partial c}{\partial S_{t}}
\end{gathered}
$$

y

$$
\xi_{c}=\frac{1}{c} \beta V_{t} \frac{\partial c}{\partial V_{t}} .
$$

Ahora bien, en virtud de (1) y (2), la ecuación de evolución de la riqueza se puede reescribir como:

$\mathrm{d} a_{t}=a_{t}\left[r+(\mu-r) x_{t}+\left(\mu_{c}-r\right) y_{t}-\frac{c_{t}}{a_{t}}\right] \mathrm{d} t+a_{t}\left(x_{t} \sigma_{t}+y_{t} \sigma_{c}\right) \mathrm{d} W_{t}+a_{t} y_{t} \xi_{c} \mathrm{~d} Z_{t}$.

En lo que sigue, la función de utilidad (satisfacción) del agente por el consumo de un bien genérico, $c_{t}$, se denotará mediante $u\left(c_{t}\right)$. Suponga que la función de utilidad indirecta, o bienestar económico, del individuo está dada por:

$$
J\left(a_{t}, V_{t}, t\right)=\max _{\left\{c_{t}, x_{t}, y_{t}\right\}} \mathrm{E}\left[\int_{\hat{t}}^{T} u\left(c_{s}\right) e^{-\delta s} \mathrm{~d} s+b\left(a_{T}, T\right) \mid \mathcal{F}_{t}\right]
$$

sujeta a las condiciones expresadas en las ecuaciones (1) y (2). El parámetro $\delta>0$ determina la tasa subjetiva de descuento del individuo, $\mathcal{F}_{t}$ denota la información relevante disponible al tiempo $t$ y $b\left(a_{T}, T\right)$ representa la función de legado (herencia o salvamento) en $T$. Observe que también $T$ representa la fecha de ejercicio de la opción. Por último, se supone que $u(\cdot)$ satisface $u^{\prime}>0$ y $u^{\prime \prime}<0$, es decir, la función de utilidad es estrictamente creciente y cóncava. En otras palabras, la utilidad marginal es positiva pero decreciente.

A continuación se emplean varias formas funcionales de la función de utilidad para obtener la ecuación diferencial parcial que caracteriza el precio de la opción.

3. Función de utilidad con coeficiente constante de aversión al riesgo En esta sección se supone que la función de utilidad tiene la siguiente forma funcional:

$$
u\left(c_{t}\right)=\frac{c_{t} \gamma}{\gamma}
$$

y que el término de legado es

$$
b\left(a_{T}, T\right)=e^{-\delta T} \frac{a_{T}^{\gamma}}{\gamma}
$$


donde $\gamma$ es el parámetro de aversión al riesgo. Observe que si $\gamma=1$ el inversionista es neutral al riesgo, mientras que si $0<\gamma<1$, el inversionista es adverso al riesgo. El caso $\gamma=0$ corresponde a la función de utilidad logarítmica, la cual se estudiará más adelante.

Para resolver el problema (7), con la función de utilidad de (8), se utilizará la ecuación de Hamilton-Jacobi-Bellman (HJB). Es decir, la función $J=J\left(a_{t}, V_{t}, t\right)$, expresada en (7), debe satisfacer la siguiente ecuación diferencial parcial de segundo orden:

$$
\begin{aligned}
0= & \max _{\left\{x_{t}, y_{t}, c_{t}\right\}}\left\{\frac{c_{t}^{\gamma}}{\gamma} e^{-\delta t}+\frac{\partial J}{\partial t}+\frac{\partial J}{\partial a_{t}} a_{t}\left[r+(\mu-r) x_{t}+\left(\mu_{c}-r\right) y_{t}-\frac{c_{t}}{a_{t}}\right]+\right. \\
+ & \frac{1}{2} \frac{\partial^{2} J}{\partial a_{t}^{2}} a_{t}^{2}\left[\left(x_{t} \sigma_{t}+y_{t} \sigma_{c}\right)^{2}+y_{t}^{2} \xi_{c}^{2}+2\left(x_{t} \sigma_{t}+y_{t} \sigma_{c}\right) y_{t} \xi_{c} \rho\right] \\
& \left.+\frac{\partial J}{\partial V_{t}} \alpha V_{t}+\frac{1}{2} \frac{\partial^{2} J}{\partial V_{t}^{2}} \beta^{2} V_{t}^{2}+\frac{\partial^{2} J}{\partial a_{t} \partial V_{t}} a_{t} \beta V_{t}\left[\left(x_{t} \sigma_{t}+y_{t} \sigma_{c}\right) \rho+y_{t} \xi_{c}\right]\right\}
\end{aligned}
$$

$\mathrm{Al}$ igualar a cero las derivadas parciales de (10) con respecto de $c_{t}, x_{t} \mathrm{y} y_{t}$, se obtienen las siguientes condiciones necesarias para un máximo:

$$
\begin{gathered}
e^{-\delta t} c_{t}{ }^{\gamma-1}-\frac{\partial J}{\partial a_{t}}=0 \\
\mu-r=-\left(x_{t} \sigma_{t}+y_{t} \sigma_{c}+y_{t} \xi_{c} \rho\right) \sigma_{t} a_{t} \frac{\frac{\partial^{2} J}{\partial a_{t}^{2}}}{\frac{\partial J}{\partial a_{t}}}-\sigma_{t} \beta V_{t} \rho \frac{\frac{\partial^{2} J}{\partial a_{t} \partial V_{t}}}{\frac{\partial J}{\partial a_{t}}}
\end{gathered}
$$

y

$$
\begin{gathered}
\mu_{c}-r=-\left[\left(y_{t} \sigma_{c}+x_{t} \sigma_{t}\right) \sigma_{c}+y_{t} \xi_{c}^{2}+\left(x_{t} \sigma_{t}+y_{t} \sigma_{c}\right) \xi_{c} \rho+y_{t} \sigma_{c} \xi_{c} \rho\right] \\
a_{t} \frac{\frac{\partial^{2} J}{\partial a_{t}^{2}}}{\frac{\partial J}{\partial a_{t}}}-\left(\rho \sigma_{c}+\xi_{c}\right) \beta V_{t} \frac{\frac{\partial^{2} J}{\partial a_{t} \partial V_{t}}}{\frac{\partial J}{\partial a_{t}}} .
\end{gathered}
$$

Se propone un candidato de solución de la forma:

$$
J\left(a_{t}, V_{t}, t\right)=e^{-\delta t} g\left(V_{t}, t\right) \frac{a_{t}^{\gamma}}{\gamma}
$$

el cual separa variables (multiplicativamente). La función $g\left(V_{t}, t\right)$ es conocida como el coeficiente del premio al riesgo. Este nombre se justifica a continuación. Observe primero que a partir de (14), se sigue que

$$
\frac{\partial J}{\partial a_{t}}=e^{-\delta t} g\left(V_{t}, t\right) a_{t}^{\gamma-1},
$$




$$
\frac{\partial^{2} J}{\partial a_{t}^{2}}=(\gamma-1) e^{-\delta t} g\left(V_{t}, t\right) a_{t}^{\gamma-2}
$$

$\mathrm{y}$

$$
\frac{\partial^{2} J}{\partial a_{t} \partial V_{t}}=e^{-\delta t} \frac{\partial g}{\partial V_{t}} a_{t}^{\gamma-1}
$$

En virtud de estas ecuaciones, el coeficiente de aversión al riesgo, el cual aparece explícitamente en (12) y (13), satisface

$$
-a_{t} \frac{\frac{\partial^{2} J}{\partial a_{t}^{2}}}{\frac{\partial J}{\partial a_{t}}}=1-\gamma=-c_{t} \frac{u^{\prime \prime}\left(c_{t}\right)}{u^{\prime}\left(c_{t}\right)}
$$

en donde $-c_{t} u^{\prime \prime}\left(c_{t}\right) / u^{\prime}\left(c_{t}\right)$ es la elasticidad de la utilidad marginal (coeficiente relativo de aversión al riesgo). Además,

$$
\frac{\frac{\partial^{2} J}{\partial a_{t} \partial V_{t}}}{\frac{\partial J}{\partial a_{t}}}=\frac{\frac{\partial g}{\partial V_{t}}}{g\left(V_{t}, t\right)}=\frac{1}{V_{t}} \frac{\partial g}{\partial V_{t}} \frac{V_{t}}{g}=\frac{1}{V_{t}} \varepsilon_{g, V}
$$

en donde $\varepsilon_{g, V}$ es la elasticidad de $g$ con respecto de $V_{t}$. Para obtener la ecuación diferencial parcial que determina el precio de la opción se requiere una solución de esquina. En particular, se requiere que haya inversión en la acción, pero no en la opción ni en el bono libre de riesgo. Al sustituir $x_{t}=1$ y $y_{t}=0$ en las ecuaciones (11), (12) y (13), éstas se transforman, respectivamente, en:

$$
\begin{gathered}
c_{t}^{\gamma-1}=g\left(V_{t}, t\right) a_{t}^{\gamma-1} \\
\mu-r=(1-\gamma) \sigma_{t}^{2}-\rho \sigma_{t} \beta V_{t} \frac{\frac{\partial g}{\partial V_{t}}}{g\left(V_{t}, t\right)}
\end{gathered}
$$

y

$$
\mu_{c}-r=(1-\gamma) \sigma_{t}\left(\sigma_{c}+\xi_{c} \rho\right)-\left(\sigma_{c} \rho+\xi_{c}\right) \beta V_{t} \frac{\frac{\partial g}{\partial V_{t}}}{g\left(V_{t}, t\right)} .
$$

En particular, los premios al riesgo para el activo subyacente y el producto derivado están dados por las ecuaciones:

$$
\lambda_{S}=\frac{\mu-r}{\sigma_{t}}=(1-\gamma) \sigma_{t}-\rho \beta V_{t} \frac{\frac{\partial g}{\partial V_{t}}}{g\left(V_{t}, t\right)}
$$

y

$$
\lambda_{c}=\frac{\mu_{c}-r}{\sigma_{c}}=\left(1+\frac{\xi_{c}}{\sigma_{c}} \rho\right)(1-\gamma) \sigma_{t}-\left(\rho+\frac{\xi_{c}}{\sigma_{c}}\right) \beta V_{t} \frac{\frac{\partial g}{\partial V_{t}}}{g\left(V_{t}, t\right)} .
$$


A partir de las ecuaciones anteriores se puede concluir que

$$
\lambda_{c}=\lambda_{S}+\frac{\xi_{c}}{\sigma_{c}} \rho(1-\gamma) \sigma_{t}-\frac{\xi_{c}}{\sigma_{c}}\left(\frac{\beta V_{t}}{g}\right) \frac{\partial g}{\partial V_{t}},
$$

la cual conduce a

$$
\begin{aligned}
& \frac{\partial c}{\partial t}+\frac{\partial c}{\partial S_{t}} r S_{t}+\frac{1}{2} V_{t} S_{t}{ }^{2} \frac{\partial^{2} c}{\partial S_{t}{ }^{2}}-r c+\left[\alpha V_{t}-\beta V_{t} \rho(1-\gamma) \sigma_{t}+\left(\frac{\beta^{2} V_{t}^{2}}{g}\right) \frac{\partial g}{\partial V_{t}}\right] \\
& \frac{\partial c}{\partial V_{t}}+\frac{1}{2} \frac{\partial^{2} c}{\partial V_{t}^{2}} \beta^{2} V_{t}^{2}+\frac{\partial^{2} c}{\partial S_{t} \partial V_{t}} \beta V_{t}^{3 / 2} S_{t} \rho=0,
\end{aligned}
$$

junto con la condición de frontera $c\left(S_{t}, V_{t}, T\right)=\max \left(S_{t}-K, 0\right)$. Asimismo, la ecuación (10) se simplifica si se sustituye el candidato de solución $J$ y la solución de esquina $x_{t}=1$ y $y_{t}=0$, en cuyo caso se obtiene

$$
\begin{aligned}
0 & =\frac{g^{\gamma /(\gamma-1)}}{\gamma}-\frac{\delta}{\gamma} g+\frac{1}{\gamma} \frac{\partial g}{\partial t}+\mu g-g^{\gamma /(\gamma-1)}+\frac{1}{2}(\gamma-1) V_{t} g \\
& +\left(\alpha V_{t}+\gamma \sigma_{t} \beta V_{t} \rho\right) \frac{1}{\gamma} \frac{\partial g}{\partial V_{t}}+\frac{1}{2}\left(\frac{\beta^{2} V_{t}^{2}}{\gamma}\right) \frac{\partial^{2} g}{\partial V_{t}^{2}} .
\end{aligned}
$$

donde se ha utilizado que

$$
c_{t}=\left[g\left(V_{t}, t\right)\right]^{1 /(\gamma-1)} a_{t} \quad \text { y } \quad \frac{\partial J}{\partial t}=\left(-\delta g+\frac{\partial g}{\partial V_{t}}\right) e^{-\delta t} \frac{a_{t}^{\gamma}}{\gamma} .
$$

De esta manera, la ecuación (20) se transforma en

$$
\begin{aligned}
& 0=-\frac{\partial g}{\partial t}+(\gamma-1) g^{\gamma /(\gamma-1)}+\left[(\delta-\mu \gamma)-\frac{1}{2} \gamma(\gamma-1) V_{t}\right] g \\
& -\left(\alpha V_{t}+\gamma \beta V_{t}^{3 / 2} \rho\right) \frac{\partial g}{\partial V_{t}}-\frac{1}{2} \beta^{2} V_{t}^{2} \frac{\partial^{2} g}{\partial V_{t}^{2}} .
\end{aligned}
$$

La condición de frontera, en este caso, es $g\left(V_{t}, T\right)=1$, lo cual asegura que se satisfaga el valor del legado en (9). Como puede observarse, se requiere la solución de (21), $g=g\left(V_{t}, t\right)$, a fin de sustituirla en (20) y poder resolver esta última en $c\left(S_{t}, V_{t}, t\right)$.

La ecuación (19) indica cómo ajustar el proceso estocástico que sigue el precio del activo subyacente, dado en la ecuación (1). En este caso,

$$
\left\{\begin{array}{l}
\mathrm{d} S_{t}=r S_{t} \mathrm{~d} t+\sigma_{t} S_{t} \mathrm{~d} W_{t} \\
\mathrm{~d} V_{t}=\left[\alpha V_{t}-\beta V_{t} \rho(1-\gamma) \sigma_{t}+\left(\frac{\beta^{2} V_{t}^{2}}{g}\right) \frac{\partial g}{\partial V_{t}}\right] \mathrm{d} t+\beta V_{t} \mathrm{~d} Z_{t}
\end{array}\right.
$$




\section{Función de utilidad logarítmica}

Considere de nuevo un consumidor racional con vida infinita maximizador de utilidad. Se supone una función de utilidad logarítmica, lo cual conduce a que el consumidor es adverso al riesgo. Como antes, se supone que el consumidor tiene acceso a un activo libre de riesgo (de incumplimiento), por ejemplo un bono cupón cero, una acción con riesgo y una opción sobre dicha acción. Se supone que la función de utilidad esperada al tiempo $t$ de un individuo representativo y competitivo tiene la siguiente forma:

$$
\mathrm{E}\left[\int_{t}^{T} \ln \left(c_{s}\right) e^{-\delta t} \mathrm{~d} s+\ln \left(a_{T}\right) \frac{e^{-\delta T}}{\delta} \mid \mathcal{F}_{t}\right],
$$

donde $\delta$ es la tasa subjetiva de descuento y $\mathcal{F}_{t}$ es la información disponible en al tiempo $t$.

El consumidor representativo posee tres diferentes activos, en términos reales: un título de deuda, de precio $b_{t}$; una acción, de precios $S_{t}$ y una opción de compra $c\left(S_{t}, t\right)$ sobre $S_{t}$. En consecuencia, la riqueza real, $a_{t}$, del individuo está dada por:

$$
a_{t}=b_{t}+S_{t}+c\left(S_{t}, t\right)
$$

Sea $x_{t}=S_{t} / a_{t}$ la proporción de la riqueza que el consumidor asigna a la tenencia de títulos de capital, $y_{t}=c / a_{t}$ la proporción de la riqueza que el consumidor asigna a la tenencia de opciones y $1-x_{t}-y_{t}=b_{t} / a_{t}$ la proporción de la riqueza que el consumidor en títulos de deuda pública. De esta manera, la evolución de la acumulación de la riqueza real sigue la ecuación diferencial estocástica de la forma

$$
\mathrm{d} a_{t}=a_{t} x_{t} \mathrm{~d} R_{S}+a_{t} y_{t} \mathrm{~d} R_{c}+a_{t}\left(1-x_{t}-y_{t}\right) \mathrm{d} R_{b}-c_{t} \mathrm{~d} t,
$$

donde $\mathrm{d} R_{S}$ es el rendimiento del activo con riesgo, $\mathrm{d} R_{c}$ es el rendimiento de la opción y $\mathrm{d} R_{b}=r \mathrm{~d} t$ el rendimiento del bono. Es importante distinguir entre las cantidades $c=c\left(S_{t}, V_{t}, t\right)$ y $c_{t}$, la primera representa el precio de la opción y la segunda el consumo. Suponga que

$$
\mathrm{d} R_{S}=\mu \mathrm{d} t+\sigma_{t} \mathrm{~d} W_{t},
$$

donde $\mu \in \mathbb{R}, \sigma_{t} \geq 0$ y $\left\{W_{t}\right\}_{t \geq 0}$ un movimiento Browniano definido sobre un espacio fijo de probabilidad equipado con una filtración $\left(\Omega^{W}, \mathcal{F}^{W},\left\{\mathcal{F}_{t}^{W}\right\}_{t \geq 0}, \mathbb{P}^{W}\right)$ y

$$
\mathrm{d} V_{t}=\alpha V_{t} \mathrm{~d} t+\beta V_{t} \mathrm{~d} Z_{t},
$$

donde $V_{t}=\sigma_{t}^{2}, \alpha>0, \beta>0$ y $\left\{Z_{t}\right\}_{t \geq 0}$ un movimiento Browniano definido sobre un espacio fijo de probabilidad equipado con una filtración $\left(\Omega^{z}, \mathcal{F}^{Z}\right.$, $\left\{\mathcal{F}_{t}^{z}\right\}_{t \geq 0}, \mathbb{P}^{z}$ ). Suponga que

$$
\operatorname{Cov}\left(\mathrm{d} W_{t}, \mathrm{~d} Z_{t}\right)=\rho \mathrm{d} t .
$$

Durante el intervalo de tiempo $[t, t+\mathrm{d} t]$, el activo subyacente cambia de $S_{t}$ a $S_{t}+\mathrm{d} S_{t}$, en consecuencia, el precio de la opción cambia de $c\left(S_{t}, V_{t}, t\right)$ a $c+\mathrm{d} c$. 
El cambio marginal en el precio de la opción se obtiene mediante el lema de Itô, como:

$$
\begin{aligned}
& \mathrm{d} c= \\
& \left(\frac{\partial c}{\partial t}+\frac{\partial c}{\partial S_{t}} \mu S_{t}+\frac{\partial c}{\partial V_{t}} \alpha V_{t}+\frac{1}{2} \sigma_{t}^{2} S_{t}^{2} \frac{\partial^{2} c}{\partial S_{t}^{2}}+\frac{1}{2} \beta^{2} V_{t}^{2} \frac{\partial^{2} c}{\partial V_{t}^{2}}+\sigma_{t} \beta S_{t} V_{t} \rho \frac{\partial^{2} c}{\partial S_{t} V_{t}}\right) \mathrm{d} t \\
& +\frac{\partial c}{\partial S_{t}} \sigma_{t} S_{t} \mathrm{~d} W_{t}+\frac{\partial c}{\partial V_{t}} \beta V_{t} \mathrm{~d} Z_{t}
\end{aligned}
$$

ó

$$
\mathrm{d} c=\mu_{c} c \mathrm{~d} t+\sigma_{c} c \mathrm{~d} W_{t}+\xi_{c} c \mathrm{~d} Z_{t}
$$

donde

$$
\begin{gathered}
\mu_{c}=\left(\frac{\partial c}{\partial t}+\frac{\partial c}{\partial S_{t}} \mu S_{t}+\frac{\partial c}{\partial V_{t}} \alpha V_{t}+\frac{1}{2} \sigma_{t}^{2} S_{t}^{2} \frac{\partial^{2} c}{\partial S_{t}^{2}}+\frac{1}{2} \beta^{2} V_{t}^{2} \frac{\partial^{2} c}{\partial V_{t}^{2}}+\sigma_{t} \beta S_{t} V_{t} \rho \frac{\partial^{2} c}{\partial S_{t} V_{t}}\right) / c \\
\sigma_{c}=\frac{\partial c}{\partial S_{t}} \frac{\sigma_{t} S_{t}}{c}
\end{gathered}
$$

y

$$
\xi_{c}=\frac{\partial c}{\partial V_{t}} \frac{\beta V_{t}}{c}
$$

Si se sustituyen las ecuaciones (25) y (27) en (24), se tiene que

$$
\begin{aligned}
\mathrm{d} a_{t} & =a_{t}\left[r+(\mu-r) x_{t}+\left(\mu_{c}-r\right) y_{t}-\frac{c_{t}}{a_{t}}\right] \mathrm{d} t+ \\
& +a_{t}\left[\left(\sigma_{t} x_{t}+\sigma_{c} y_{t}\right) \mathrm{d} W_{t}+y_{t} \xi_{c} \mathrm{~d} Z_{t}\right] .
\end{aligned}
$$

Sea

$$
J\left(a_{t}, V_{t}, t\right)=\max _{\left\{c_{t}, x_{t}, y_{i}\right\}} \mathrm{E}\left[\int_{t}^{T} \ln \left(c_{s}\right) e^{-\delta t} \mathrm{~d} s+\ln \left(a_{T}\right) \frac{e^{-\delta T}}{\delta} \mid \mathcal{F}_{t}\right]
$$

La condición necesaria del problema de control óptimo estocástico en el que el consumidor racional desea maximizar la utilidad total queda expresado como:

$$
\begin{aligned}
0= & \ln \left(c_{t}\right) e^{-\delta t}+J_{t}+J_{a} a_{t}\left[r+(\mu-r) x_{t}+\left(\mu_{c}-r\right) y_{t}-\frac{c_{t}}{a_{t}}\right] \\
& +\frac{1}{2} J_{a a} a_{t}^{2}\left[\left(\sigma_{t} x_{t}+\sigma_{c} y_{t}\right)^{2}+\xi_{c}^{2} y_{t}^{2}+2\left(\sigma_{t} x_{t}+\sigma_{c} y_{t}\right) \xi_{c} y_{t} \rho\right] \\
& +J_{V} \alpha V_{t}+\frac{1}{2} J_{V V} \beta^{2} V_{t}^{2}+J_{a V} a_{t} V_{t} \beta\left[\left(\sigma_{t} x_{t}+\sigma_{c} y_{t}\right) \rho+\xi_{c} y_{t}\right] .
\end{aligned}
$$

Considere el siguiente candidato de solución

$$
J\left(a_{t}, V_{t}, t\right)=\left[\ln \left(a_{t}\right)+g\left(V_{t}, t\right)\right] \frac{1}{\delta} e^{-\delta t} .
$$


En este caso, se sigue que $g\left(V_{t}, T\right)=0$. Asimismo,

$$
\begin{aligned}
0= & \ln \left(c_{t}\right)-\left[\ln \left(a_{t}\right)+g\left(V_{t}, t\right)\right]+\frac{1}{\delta} \frac{\partial g}{\partial t}+\frac{1}{\delta}\left[r+(\mu-r) x_{t}+\left(\mu_{c}-r\right) y_{t}-\frac{c_{t}}{a_{t}}\right] \\
& -\frac{1}{2 \delta}\left[\left(\sigma_{t} x_{t}+\sigma_{c} y_{t}\right)^{2}+\xi_{c}^{2} y_{t}^{2}+2\left(\sigma_{t} x_{t}+\sigma_{c} y_{t}\right) \xi_{c} y_{t} \rho\right]+ \\
& +\frac{\alpha}{\delta} \frac{\partial g}{\partial V_{t}} V_{t}+\frac{\beta^{2}}{2 \delta} \frac{\partial^{2} g}{\partial V_{t}^{2}} V_{t}^{2} .
\end{aligned}
$$

Las condiciones de primer orden son

$$
\begin{gathered}
c_{t}=\delta a_{t}, \\
\mu-r=\left(\sigma_{t} x_{t}+\sigma_{c} y_{t}\right) \sigma_{t}+\xi_{c} y_{t} \sigma_{t} \rho
\end{gathered}
$$

$\mathrm{y}$

$$
\mu_{c}-r=\left(\sigma_{t} x_{t}+\sigma_{c} y_{t}\right) \sigma_{c}+y_{t} \xi_{c}^{2}+\left(\sigma_{t} x_{t}+\sigma_{c} y_{t}\right) \xi_{c} \rho+y_{t} \sigma_{c} \xi_{c} \rho .
$$

Es fácil verificar que $\beta=1 / \delta$. Las dos últimas condiciones se pueden reescribir como

$$
\lambda_{S} \equiv \frac{\mu-r}{\sigma_{t}}=\sigma_{t} x_{t}+\sigma_{c} y_{t}+\xi_{c} y_{t} \rho
$$

$\mathrm{y}$

$$
\lambda_{c} \equiv \frac{\mu_{c}-r}{\sigma_{c}}=\sigma_{t} x_{t}+\sigma_{c} y_{t}+y_{t} \frac{\xi_{c}^{2}}{\sigma_{c}}+\left(\sigma_{t} x_{t}+\sigma_{c} y_{t}\right) \rho \frac{\xi_{c}}{\sigma_{c}}+y_{t} \xi_{c} \rho .
$$

Al sustituir (33) en la expresión anterior, se tiene que

$$
\lambda_{c}=\lambda_{S}\left(1+\rho \frac{\xi_{c}}{\sigma_{c}}\right)+y_{t} \frac{\xi_{c}^{2}}{\sigma_{c}}\left(1-\rho^{2}\right) .
$$

Si $y_{t}=0$, entonces

$$
\lambda_{c}=\lambda_{S}\left(1+\rho \frac{\xi_{c}}{\sigma_{c}}\right) .
$$

Después de sustituir (27), (28) y (29) en la ecuación anterior, se obtiene

$$
\begin{aligned}
& \frac{\partial c}{\partial t}+\frac{\partial c}{\partial S_{t}} r S_{t}+\frac{1}{2} \frac{\partial^{2} c}{\partial S_{t}^{2}} V_{t} S_{t}^{2}-r c+(\alpha-\lambda \beta) \frac{\partial c}{\partial V_{t}} V_{t}+ \\
& +\frac{1}{2} \beta^{2} V_{t}^{2} \frac{\partial^{2} c}{\partial V_{t}^{2}}+\frac{\partial^{2} c}{\partial S_{t} V_{t}} \beta S_{t} V_{t}^{3 / 2} \rho=0
\end{aligned}
$$

donde

$$
\lambda \equiv \lambda_{S} \rho .
$$

Si se sustituyen $x_{t}=1$ y $y_{t}=0$ en la condición HJB, se tiene que

$$
0=\log (\delta)-g+\frac{1}{\delta} \frac{\partial g}{\partial t}+\frac{\mu}{\delta}-1-\frac{1}{2 \delta} \sigma_{t}^{2}+\frac{\alpha}{\delta} \frac{\partial g}{\partial V_{t}} V_{t}+\frac{1}{2 \delta} \frac{\partial^{2} g}{\partial V_{t}^{2}} \beta^{2} V_{t}^{2}
$$


Ahora bien, en virtud de (33), se sigue que $\sigma_{t}^{2}=\mu-r$. De esta manera,

$$
0=\delta[\log (\delta)-1]+\frac{1}{2}(\mu+r)-\delta g+\frac{\partial g}{\partial t}+\alpha \frac{\partial g}{\partial V_{t}} V_{t}+\frac{1}{2} \frac{\partial^{2} g}{\partial V_{t}^{2}} \beta^{2} V_{t}^{2}
$$

junto con la condición de frontera $g\left(V_{t}, T\right)=0$. La solución de esta ecuación diferencial parcial es independiente de $V_{t}$ y está dada por

$$
g(t)=A-A e^{-\delta(T-t)}
$$

donde

$$
A=\log (\delta)-1+\frac{1}{2 \delta}(\mu+r)
$$

Observe que $g$ satisface

$$
\frac{\mathrm{d} g}{\mathrm{~d} t}=\delta g-\delta A
$$

y $g(T)=0$

\section{Bibliografía}

Lewis, A. L. (2000). Option Valuation Under Stochastic Volatility: With Mathematica Code. Finance Press. U. K.

Kim, I. J, and S. Kim (2004). Empirical Comparison of Alternative Stochastic Volatility Option Pricing Models: Evidence from Korean KOSPI 200 Index Options Market. Pacific-Basin Finance Journal, 12(2), pp. 117-142.

Jones, C. S. (2003). The Dynamics of Stochastic Volatility: Evidence from Underlying and Options Markets. Journal of Econometrics, 116(1-2), pp. 181-224. 